Meta

Journal des traducteurs

Translators' Journal

\title{
Antonine Maillet, traduite ou trahie?
}

\section{John F. Patterson}

Volume 28, numéro 4, décembre 1983

URI : https://id.erudit.org/iderudit/003798ar

DOI : https://doi.org/10.7202/003798ar

Aller au sommaire du numéro

Éditeur(s)

Les Presses de l'Université de Montréal

ISSN

0026-0452 (imprimé)

1492-1421 (numérique)

Découvrir la revue

Citer cet article

Patterson, J. F. (1983). Antonine Maillet, traduite ou trahie? Meta, 28(4),

352-357. https://doi.org/10.7202/003798ar

Ce document est protégé par la loi sur le droit d'auteur. L'utilisation des services d'Érudit (y compris la reproduction) est assujettie à sa politique d'utilisation que vous pouvez consulter en ligne.

https://apropos.erudit.org/fr/usagers/politique-dutilisation/ 


\section{ANTONINE MAILLET, TRADUITE OU TRAHIE?}

JOHN F. PATterson

Vous savez tous quelle est l'importance d'Antonine Maillet dans le monde littéraire canadien et son grand rôle dans l'éveil de la conscience acadienne. Il va donc sans dire que celui ou celle qui entreprend de traduire un de ses ouvrages prend sur soi de lourdes responsabilités, car c'est de son travail que dépend l'idée que se fait le lecteur et de son auteur et du peuple dont elle est le porte-parole. Le traducteur s'impose en même temps une tâche assez difficile, étant donné le milieu social et linguistique où Antonine Maillet situe ses ouvrages, et son style tout particulier.

Il nous semble donc qu'avant de commencer notre examen des deux ouvrages en question, il est d'une importance capitale d'essayer d'arriver à une définition du travail du traducteur. Voici ce que nous en dit le Petit Robert :

Traduire: Faire que ce qui était énoncé dans une langue le soit dans une autre, en tendant à l'équivalence sémantique et expressive des deux énoncés.

Voyons en quelle mesure les deux traducteurs* ont accompli leur tâche. D'abord, Barbara Godard. Dans sa Tale of Don l'Orignal, elle traduit parfois assez heureusement l'humour si particulier chez Antonine Maillet. Celle-ci ayant dit du sinistre Sam à Mateur qu'il chassait «aussi bien ... la grue sans plumes que la grue à plumage » (DO 36), Madame Godard fait preuve d'imagination, et réussit à préserver l'ambiguïté de l'original : «Sam Amateur ... hunted ... the featherless bird of the street as well as the feathered one in the sky.» (TDO 25) Cette imagination peut parfois la mener assez loin. Comparons, à titre d'exemple, le passage suivant à son original :

Maillet (DO 28)

Godard (TDO 28)

Ah! mais
quoi c'est que
ces pêches,
asteur!
Un homme s'en revient
de la morue
comme des noces.
Il ne lui manque
pas un poil
pas un chicot
pas une patte.
C'est pas chrétien,
c'te pêche.

Oh! but

what's

all this finnin and sinnin

these days!

A man comes home

from cod and whores

like he's been to a weddin.

not missin

a hair

nor a stump of a tooth

nor a paw, neither.

It ain't Christian,

this fishin ain't,

lemme tellya.

* Barbara Godard, (tr.) The Tale of Don l'Orignal, Toronto/Vancouver, 1978, Clarke, Irwin and Company Ltd. (TDO)

Luis de Céspedes, (tr.) La Sagouine, Toronto, 1979, Simon \& Pierre Publishing Company Limited. (C) 
Ce passage est instructif, car on y voit des traits typiques de The Tale of Don l'Orignal. La traductrice s'éloigne, parfois considérablement, de l'original : «finnin and sinnin» «cod and whores», «lemme tellya». Par contre, assez souvent elle le suit de très près : "It ain't Christian» dans le contexte, ne rendant pas «c'est pas chrétien.» Ce trait donne un certain petit goût français à la traduction. En plus, et surtout, le niveau de langage n'est pas celui du texte de Maillet : «lemme tellya», les deux «ain't». On peut en dire autant, en général, de tous les dialogues du livre.

Les libertés que la traductrice se permet se voient peut-être mieux dans le passage qui traite de la résurrection miraculeuse de Citrouille, le «mal nayé »: là où Antonine Maillet décrit son héros (DO 93) «s'attrapant les côtes dans un suprême effort pour s'accrocher à la terre ...» (il va rendre sa gorge), $\mathbf{M}^{\text {me }}$ Godard le dépeint ainsi : «hitting the shore in a supreme effort to cling to the earth, the shipwrecked man gave back to the island all the silly herbs the learned naturalist had stuffed him with.» (TDO 70) Ma foi, oui, dans un tel contexte, quoi de plus à propos que «silly herbs»?

Quant au phénomène contraire, bien plus courant dans cette traduction, et qui consiste à rester très près, par la forme du texte original, considérons le passage où Maillet parle de la chapelière, qui jalouse toutes les femmes jolies, et deux en particulier... Voici les paroles de $\mathbf{M}^{\text {me }}$ Godard: «the milliner, not forgiving these two women their heads...» (TDO 92) Les exemples de cette espèce sont nombreux: "Our mayor had made rain and sunshine», (TDO 105), «There wasn't a cat left in Victory Square», (TDO 44) "Ya liked them girls from the grand world too much» (TDO 64), et nous en passons...

Or, ces deux phénomènes, qui semblent à première vue opposés, sont deux faces différentes d'un seul problème. Cette traduction est très inégale, et si parfois Barbara Godard réussit admirablement à traduire à la fois la pensée et le style d'Antonine Maillet, d'autres fois, comme nous venons de voir, la réussite n'est pas totale. Il nous semble que cela se doit au fait que souvent elle reste à la surface, traduisant les mots plutôt que les idées, ce qui parfois lui fait perdre le fil d'un passage. Examinons ensemble le passage suivant. Nous avons mis en parallèle ici l'original (au milieu), la traduction de Godard (à gauche) et notre propre suggestion (à droite).

TDO 29

«Sluts, » said la S

«That's a fisherman's life,» answered a bum.

«Slut of a fish, too

replied the heroic woman.

«Still them sluts

gave la Sagouine

all her men, »

Noume let fly.
DO $20-21$

- Des saloppes, dit la $\mathbf{S}$. - C'est la vie de pêche, répondit un chemineau.

- Saloppe de pêche aussi, reprit l'héroique femme. - C'est pourtant ces saloppes-là qui ont fourni à la Sagouine tous ses hommes, lui lança Noume.
JP

«Bitches, » said la S

"That's the fishing life," answered a bum.

«Fishing's a bitch, too", replied the heroic woman.

Still those bitches gave la Sagouine all her men,»

Noume snapped back...

La traduction de ce passage par Barbara Godard, c'est l'évidence même, prête à confusion. Quel sens attribuer, dans le contexte, à «slut of a fish»? 
La difficulté, évidemment, provient du fait que le terme slut ne s'emploie pas de cette façon en anglais, et que pêche traduit fishing plutôt que fish.

Le trait le plus frappant de cette traduction reste, cependant, le langage des dialogues. Or, celui de Maillet est essentiellement le même qu'elle emploie dans la Sagouine. Dans son article «La Langue de la Sagouine»publié dans l'édition Leméac du livre en 1974, André Belleau affirme qu'il s'agit d'une langue traditionnelle, d'un français régional apporté de Touraine par les ancêtres des Acadiens modernes. On peut objecter que de simples pêcheurs n'auraient pas parlé une langue aussi soignée, mais c'est néanmoins celle qu'Antonine Maillet, leur créatrice, a choisi de leur faire parler.

C'est apparemment dans le souci de faire parler «peuple» ses personnages (souci que l'auteur de l'original ne partage pas autrement) que Barbara Godard charge son texte d'incorrections grammaticales, de prononciations défectueuses et de blasphèmes, qui le plus souvent ne correspondent à rien dans le texte d'Antonine Maillet. You se dit ya, et your, yer. L'interjection lemme tellya apparaît trois fois, tout à fait gratuitement. Le comble, dans le genre, c'est le moment où la Sagouine prie pour le salut de son île menacée, (TDO 35) en commençant ainsi : "God the Father, it's ya I'm talkin to." Or, le dernier emploi imaginable de $y a$, c'est sûrement comme pronom tonique. Le pronom them, lui, remplace souvent l'article : «Ç'a rien de drôle, une guerre», «ain't nothin funny about them wars»; «toutes les grosses têtes», «all them bigwigs»; ou bien, il apparaît tout gratuitement : «beaucoup d'huîtres», «lotsa them oysters.» Because se dit toujours 'cause'. Le désaccord entre sujet et verbe est courant.

Nous avons déjà signalé la tendance chez Barbara Godard d'ajouter des éléments au texte, comme ses lemme tellya et ses them. Le meilleur exemple de cette inflation linguistique est sans doute le suivant : «Where the devil godalmighty bejeezus tarnation is Citrouille!» (TDO 41). Ce qui a déclenché ce flot de blasphèmes, c'est cette innocente petite phrase: "Où c'est qu'est Citrouille?» (TDO 41) Côté blasphèmes, elle va jusqu'à prêter à la Sainte, personnage archidévot s'il en fût, comme l'indiquent son nom et le texte de roman, l'exclamation «Christ!»

Autre élément gratuit du langage, peu réaliste dans le contexte, c'est l'introduction dans le texte d'un certain argot moderne courant dans les grands centres urbains nord-américains. Une belle fille, c'est «a pretty chick», «la grosse affaire», «the big deal»; «il a un cœur de poule», «he's chicken»; «une créature», c'est «a dame».

Que dire, en somme, du dialogue de The Tale of Don l'Orignal? Plutôt que traduction, on devrait peut-être parler de remaniement, d'adaptation, terme que l'on pourrait appliquer également à certains passages narratifs, comme nous venons de le voir. C'est une considération d'autant plus importante que l'on va voir se reproduire les mêmes phénomènes dans la traduction de la Sagouine de Luis de Céspedes. Mais là, cela devient plus grave, du fait qu'il s'agit d'un monologue. Voici quelques exemples de l'anglais de Céspedes. Là où Maillet avait écrit : «ils vouliont rien comprendre, les enfants, ( $M$ 73), lui met : «they didn' wanna und'stand not'n, 'em kids». (C 40) (Notons que Cés- 
pedes fait le même emploi de 'em que fait Barbara Godard de them.) Autre exemple: «J'avons rien à nous autres» (M 111) devient «We got not'n ours.» (C 83)

Ses choix lexicaux sont pour beaucoup dans le niveau du langage de Céspedes. «un mort» (M 144), «a stiff» (C 115); «un enfant» (M 144), «a kid» (C 115). Comme Barbara Godard encore, il enrichit le texte de blasphèmes et de scatologie gratuits. Ainsi, «le frolic (la fête) allait commencer» (M 125) se traduit «all hell was gonna break loose» (C 95). Tel personnage qui dans le texte de Maillet «savait vous faire trembler de peur» (M 141) «knew how to scare the shit out of you».

Céspedes, lui aussi, affectionne l'argot urbain moderne. C'est ainsi qu'il traduit l'exclamation archi-acadienne «T'as qu'à ouère ! par « Better believe it!» (C 28). Un certain individu faillit «chavirer» chez Maillet; chez Céspedes, « he come pretty close to losin his marbles ». Il n'hésite même pas à employer, à plusieurs reprises, l'expression hippie, totalement gratuite, «like». («Like, almost all the parish was against it.») (C 93)

Cela n'empêche en rien, pourtant, que cette traduction ne sente le français, au moins autant que celle de Barbara Godard. «them ones, if only I could tell you» (C 32); "all your sins and all the ones of your man 'n all the ones of your children" (C 74); "The priest, him, when he preaches" (C 73); «Us, we ain't never seen...» (C 73); "Ah, fer a rectory, that sure was a rectory » (C 73). Dans le chapitre «Les Cartes», il s'agit des cartes comme indicatrices du destin. Or, Antonine Maillet nous prévient, par la bouche de sa Sagouine : «jetez... vos yeux du côté du treufle» (M 162), et Céspedes de traduire : «keep an eye on the side of that club.» (C 133). Au Japon, après la bombe atomique, «Not one cat came out of there alive.» (C 87) Frank à Thiophie «flung his dentures down the drain and stayed with a hole in his face.» (C 61) («drain» traduit, apparemment, le mot puits de l'original.) «Ils pretendont que c'est pus la mode " (M 54) devient «They pr'tend it ain't done no more.» (C 19), et «ta parouesse actuelle» (M 141), «yer actual parish». Parlant d'une certaine maladie qui afflige les jeunes et les moins jeunes, au printemps, Antonine Maillet fait dire à la Sagouine: "C'est pas un mal de cœur», (M 176) que Céspedes traduit bien gentiment: "It ain't a heart-ache.» Il avait besoin de bons amis, M. Céspedes. Ce ne sont pas les faux qui lui manquent.

Par contre, comme Barbara Godard, $M$. de Céspedes se permet certaines libertés vis-à-vis de son original. C'est ainsi que «la belle robe de dentelle " que porte l'enfant Jésus dans la procession de Noël se transforme par miracle en «his beatiful (sic) face». (C 41) Et il y a plus miraculeux encore: "The good ol' Sainte, in the flesh, body and soul, bones ' $\mathrm{n}$ all, she stretched almost all the way to the altar.» (C 95) Cela a été inspiré, selon toute apparence, par le passage suivant: "La Sainte, en chair et en os, corps et âme, tripes et boyaux, s'a avancé de tout son long jusqu'à la sainte table.» (M 124) Les fermiers en carême, ne pouvant se procurer de poisson, «mangiont du pain pis de la mélasse pour pas mouri' avec du lard salé sus la conscience.» (M 154) Cependant, dans le texte anglais ils ont encore moins de chance: "They would eat bread and molasses to survive with salt pork on their conscience.» (C 125) Au cours 
du recensement, «ils te questiounont... ton nom, tous tes noms de baptême, ton père, ta mère, ta darnière maladie.» (M 190) Or, cette «darnière maladie» devient chez $M$. de Céspedes «yer terminal sickness»! (C 163)

Qu'est-ce qui ressort, en fin de compte, des libertés que prennent ces deux traducteurs? Eh bien, il en ressort, à notre avis, que ce qui constitue l'originalité d'Antonine Maillet se perd souvent. Pour commencer, ce que l'on pourrait appeler couleur locale, que cela tienne à un certain tour de phrase, à un certain vocabulaire, ou à certaines allusions, tend à disparaître, surtout chez Céspedes. Le verbe greyer, avec sa résonance maritime, s'emploie souvent dans les textes d'Antonine Maillet, et le plus souvent comme synonyme d'habiller. Céspedes traduit «J'étions pas assez ben greyés» par "we wasn' dressed good enough». (C 70) Dorée se traduit par boat. (Barbara Godard, elle, avait traduit drageur par dredger, au lieu de dragger.) Les Indiens de Céspedes, eux, habitent des wigwams au lieu des prosaïques cabanes des Micmacs du livre de Maillet. «Arvin's», ça doit sûrement être le magasin des célèbres Irving à Bouctouche, mais il faut le savoir.

Ce qu'il y a peut-être de plus désolant, c'est que souvent l'humour, trait si caractéristique des ouvrages d'Antonine Maillet, ne passe pas par le filtre de la traduction. Par exemple, dans Don l'Orignal il est question, à un moment donné, d'une "Indienne mère, assez sorcière de son métier». Or, «an Indian mother, skilled in witchcraft», c'est beau, si l'on veut, mais ce n'est pas du Maillet. («An Indian mother, a bit of a witch by trade» aurait peut-être mieux fait l'affaire.) Tout à la fin du livre, surgit «un tiers monde, vigoureux et hardi" (DO 144). La traduction de Barbara Godard, "a hardy and vigorous developing world" nous semble plutôt insuffisante du fait que, effectivement, il s'agit littéralement d'un troisième monde. Quand, dans la Sagouine cette héroïne se lamente que «Un Acadjen, c'est point une nationalité, par rapport que c'est pas écrit dans les livres de Jos Graphie», M. de Céspedes, lui, parle de «Joe Graphy's books». Et quand elle déclare, avec nostalgie, : «J'ai été jeune dans ma jeunesse, moi itou», il nous donne pour équivalent "I was young when I was young»!

Vous étonnerait-il de savoir que l'ouvrage de M. de Céspedes a été subventionné par le Conseil des Arts? Ça fait jongler, ça.

Que faut-il conclure, en somme, sur la valeur de ces deux ouvrages comme traductions, c'est-à-dire comme clés à l'auteur et à son monde? Tout simplement qu'Antonine Maillet - et son public anglophone - méritaient mieux. En toute franchise, il y aurait autre chose à dire sur l'ouvrage de M. de Céspedes. Si celui de Barbara Godard nécessite des révisions considérables, celui de Céspedes est à refaire à partir de zéro. Vous avez pu vous apercevoir qu'il y a autre chose dans son style anglais qu'un choix de niveau de langage. Disons-le : il n'a pas la maitrise de la langue anglaise qui lui aurait permis de s'exécuter convenablement.

Il y a pourtant une lueur à l'horizon. La traduction par M. Philip Stratford de Pélagie-la-Charrette, l'ouvrage qui a valu à Antonine Maillet le Prix Goncourt, semblerait nettement supérieure à celles que nous venons d'examiner. Nous ne pouvons nous vanter de l'avoir étudiée en détail, mais un premier 
coup d'œil semblerait indiquer que ce traducteur a su rendre accessible au public de langue anglaise, dans la mesure du possible, la pensée et l'art d'Antonine Maillet. 